INPLASY PROTOCOL

To cite: Pentapati et al. Diagnostic accuracy of ultrasound imaging for the detection of facial fractures systematic review and metaanalysis. Inplasy protocol 2020120064. doi: 10.37766/inplasy2020.12.0064

Received: 12 December 2020

Published: 12 December 2020

Corresponding author: Kalyana Pentapati

drkalyan81@gmail.com

Author Affiliation:

Manipal College of Dental Sciences, Manipal, Manipal Academy of Higher Education, Manipal, Udupi, Karnataka, India

Support: Nil.

Review Stage at time of this submission: Preliminary searches.

Conflicts of interest: Nil.

\section{Diagnostic accuracy of ultrasound imaging for the detection of facial fractures - systematic review and meta-analysis}

Pentapati, K; Gadicherla, S²; Singh, A3; Smriti, K4․

Review question / Objective: Ultrasound imaging has been used in the diagnosis of facial fractures. We wish to evaluate the diagnostic accuracy of ultrasound imaging for the detection of facial fractures. P: Patients with suspected facial fractures would be included; I: Ultrasound Imaging or Ultrasonography; C: Computed tomography scan or Cone Beam Computed Tomography (CBCT) scan.

Condition being studied: Facial fractures.

Information sources: Pubmed, Scopus, CINAHL, Web of Science, Dentistry and Oral Sciences source (EBSCO host) will be searched from inception to 12 september 2020. The references from the selected studies would be manually searched. Only studies in English would be included.

INPLASY registration number: This protocol was registered with the International Platform of Registered Systematic Review and Meta-Analysis Protocols (INPLASY) on 12 December 2020 and was last updated on 12 December 2020 (registration number INPLASY2020120064).

\section{INTRODUCTION}

Review question / Objective: Ultrasound imaging has been used in the diagnosis of facial fractures. We wish to evaluate the diagnostic accuracy of ultrasound imaging for the detection of facial fractures. P: Patients with suspected facial fractures would be included; I: Ultrasound Imaging or UItrasonography ; C : Computed tomography scan or Cone Beam Computed Tomography (CBCT) scan. 
Rationale: Ultrasound imaging is a low cost, readily available, rapid, dynamic, noninvasive, real-time imaging, reproducible, safe, no radiation exposure, and easy to use imaging modality for the head and neck region. It is a suitable alternative for CT scans. This review evaluates the diagnostic accuracy of ultrasound imaging for identifying facial fractures.

Condition being studied: Facial fractures.

\section{METHODS}

Search strategy: Search terms: UItrasonography OR UItrasound Tomography, X-Ray Computed OR CT Scan OR Computed Tomography OR CBCT OR cone beam computed tomography Mandible Fracture OR Mandibular Fracture OR Zygoma Fracture OR Facial Fracture OR Zygomatic Fracture OR Zygomatic arch Fracture OR Orbit Fracture OR Orbital Fracture OR Maxilla Fractures OR Maxillary Fractures OR Nasal fracture OR Nasal Bone/injuries Comparison OR Diagnosis OR Diagnostic OR Sensitivity OR Specificity OR Accuracy.

Participant or population: Patients with suspected facial fractures.

Intervention: Ultrasound Imaging or Ultrasonography.

Comparator: Computed tomography scan or Cone Beam Computed Tomography (CBCT) scan.

Study designs to be included: Randomized trials, diagnostic accuracy studies.

Eligibility criteria: Studies done on humans, reported in English were included. Studies which report sensitivity and specificity or true positive, false positive, true negative, false negative values or studies in which these values can be calculated were included.

Information sources: Pubmed, Scopus, CINAHL, Web of Science, Dentistry and Oral Sciences source (EBSCO host) will be searched from inception to 12 september
2020. The references from the selected studies would be manually searched. Only studies in English would be included.

Main outcome(s): This review will evaluate the diagnostic accuracy of ultrasound imaging of facial fractures (Sensitivity, specificity, positive and negative likelihood ratio and diagnostic odds ratio).

Data management: A list of studies obtained from searching the various databases would be imported to a webbased software (Rayyan). Title and abstract screening will be done by two review authors independently. Full-text screening of eligible studies will be done by two review authors independently. The data extraction would be done by two review authors independently. Discrepancies will be resolved by a third review author.

Quality assessment / Risk of bias analysis: Quality assessment of Diagnostic Accuracy studies - 2 (QUADAS - 2) would be used. Risk of bias would be performed by two review authors independently.

Strategy of data synthesis: Meta-analysis will be performed on the data extracted from various studies (TP, FP, TN and FN) using MetaDTA software and Summary receiver operating characteristic (SROC) curve would be plotted. Sensitivity analysis: Sensitivity analysis would be performed for the included studies by excluding low quality studies to evaluate the robustness of the model. Subgroup analysis: Subgroup analysis would be performed based on the study characteristics (risk of bias, study design) and patient characteristics (site of fractures).

Subgroup analysis: Subgroup analysis would be performed based on the study characteristics (risk of bias, study design) and patient characteristics (site of fractures).

Sensibility analysis: Sensitivity analysis would be performed for the included studies by excluding low quality studies to evaluate the robustness of the model. 
Language: Publications in English language would be included.

Country(ies) involved: India.

Keywords: Ultrasound, Computed tomography, fracture, diagnosis, accuracy, sensitivity, specficity.

Dissemination plans: We plan to publish this review in academic journals.

Contributions of each author:

Author 1 - Kalyana Pentapati - Ideation, literature search, screening, analysis, final draft.

Email: drkalyan81@gmail.com

Author 2 - Srikanth Gadicherla - screening, data extraction, initial draft.

Email: gadi_mds@rediffmail.com

Author 3 - Anupam Singh - data extraction, risk of bias assessment, initial draft.

Email: anupam.singh@manipal.edu

Author 4 - Komal Smriti - screening, data extraction, risk of bias assessment, initial draft.

Email: komalmds1@gmail.com 